\title{
Subkutane Flüssigkeitssubstitution bei betagten Patienten während Hitzewellen
}

Joachim Schnürle

Alte Patienten nehmen häufig nicht ausreichend Flüssigkeit zu sich - ein Problem, mit dem angesichts zunehmender Hitzeperioden in Zukunft häufiger zu rechnen sein wird. Die einfachste Maßnahme - mehr Trinken - stößt bei alten Patienten jedoch oft an Grenzen. Dieser Beitrag schildert eine effiziente Alternative, die auch von geschulten Laien durchgeführt werden kann: die subkutane Flüssigkeitssubstitution.

Hitzewellen und Mortalität | Während des „Jahrhundertsommers“ 2003 wurde für Deutschland eine Übersterblichkeit von 7000 Personen beschrieben [39]. Betroffen waren insbesondere ältere und bettlägrige Patienten. Auch in den Folgejahren kam es zu längeren Hitzeperioden. Auf verschiedenen Ebenen der öffentlichen Gesundheitsfürsorge wurde dieses Problem wahrgenommen und evaluiert. So beschäftigten sich das Bundesgesundheitsministerium und die WHO mit den Folgen von Hitzewellen in Deutschland und Europa [11, 40]. Auch in internationalen Publikumszeitschriften ist ein Problembewusstsein entstanden (z.B. Newsweek: „Are you ready for more?" [3]). In der Zwischenzeit wurde vom Deutschen Wetterdienst (DWI) ein Hitzewarnsystem etabliert.

Klimawandel-Projektionen prognostizieren häufigere, intensivere und länger andauernde Hitzewellen - auch in Nordeuropa [23].

Eingeschränkte Adaptation | Unter Hitzewellen leiden vermehrt vulnerable Personengruppen wie Säuglinge und ältere Menschen, insbesondere bei zusätzlichen Begleiterkrankungen [21]. Oft wird eine sogenannte Trinkschwäche diagnostiziert. Die Ursachen sind mannigfaltig, z.B.

- eine dementielle Problematik,

- eine eingeschränkte Verfügbarkeit von Getränken oder

- das abnehmende Durstgefühl [28].

Nicht selten kommt dazu eine Angst vor Inkontinenz oder nächtlichen Toilettengängen, die zu einer verminderten Flüssigkeitsaufnahme führen $[15,32]$.

Mit zunehmendem Alter sinkt die Toleranz des Organismus gegenüber Wasser- und Elektrolytveränderungen.

Physiologische Veränderungen I Grund für die verminderte Kompensationsfähigkeit älterer Patienten sind physiologische Alterungsprozesse.
- Bei Hitzeperioden steigt die Perspiratio insensibilis von $300-500 \mathrm{ml}$ auf bis zu $10 \mathrm{l}$ pro Tag unter tropischen Arbeitsbedingungen [41].

- Im Alter sind Schweißsekretion und kardiovaskuläre Funktion bei Hitzeexposition allerdings verändert $[14,16]$.

- Der Fettanteil an der Körpermasse nimmt zu und das Gesamtkörperwasser sinkt um 10\%. Dadurch ist die intrazelluläre Flüssigkeitshomöostase anfälliger für eine verminderte $\mathrm{Zu}$ fuhr bzw. einen erhöhten Verlust von Flüssigkeit.

- Die Sekretion von ADH und ANP ist Alterungsvorgängen unterworfen, die letzlich das Durstgefühl reduzieren. Bildung und Ausschüttung von ADH können zudem beeinflusst werden von

- Sulfonylharnstoffen,

- Diuretika,

> ischämischen Ereignissen,

- entzündliche Veränderungen oder

> paraneoplastischen Vorgängen [32].

Komplikation durch Medikamente | Ältere Menschen sind aufgrund von Komorbiditäten häufig auf Medikamente angewiesen, die eine Exsikkose provozieren können. Hierzu gehören:

- ACE-Hemmer,

- Diuretika,

- Laxantien.

Durch sedierende Nachtmedikation, z. B. Benzodiazepine, kommt es zu einer vermehrten Tagesschläfrigkeit und in der Folge zur verminderten oralen Flüssigkeitsaufnahme [32].

Bei Hitzeperioden sind ältere Menschen anfälliger für Dehydratationszustände, insbesondere bei febrilen Begleiterkrankungen und Polypharmazie.

\section{Diagnostik}

Exsikkose oder Volumenmangel? I Die beiden Krankheitsbilder unterscheiden sich pathophysiologisch [5, 37]: 
- Exsikkose=primär intrazellulärer Mangelzustand (hypertone Dehydratation).

- Volumenmangel=extrazelluläres Defizit, das sich intravaskulär manifestiert (hypotone oder isotone Dehydratation).

Diagnosekriterien | Durch Medienberichte, Kampagnen und Faltblätter hat die öffentliche Wahrnehmung von Hitze als Gesundheitsproblem seit 2003 deutlich zugenommen. Bei Pflegepersonal besteht jedoch weiterhin Schulungsbedarf, um Dehydratationszustände zu erkennen. Bei einer Exsikkose gibt es typische klinische Zeichen:

- stehende Hautfalten,

- trockene Schleimhäute,

- Orthostase/Kollaps,

- Tachykardie,

- Durstgefühl,

- ein subakut bis akut auftretendes Delir,

- zunehmende Lethargie und Desorientierung,

- sowie unspezifische Hinweise wie

$\checkmark$ Müdigkeit,

- Kopfschmerzen,

v verminderte Miktionsfrequenz,

$\checkmark$ dunkler Urin.

Eine Exsikkose ist anzunehmen bei einem Gewichtsverlust von mehr als $3 \%$ in wenigen Tagen. Hypohydratation ist eine häufige Ursache von deliranten Zuständen [19, 34].

Klinische Diagnostik bei alten Patienten |Bei alten Patienten müssen allerdings stets die physiologischen Veränderungen berücksichtigt werden:

- Das Durstgefühl ist oft altersbedingt vermindert.

- Die Schleimhäute wirken bei bettlägrigen Menschen mit Mundatmung auch bei guter Hydratation häufig trocken.

- Bei vermindertem subkutanen Fett sind Hautfalten regelmäßig provozierbar, unabhängig vom Flüssigkeitshaushalt $[21,44]$.

Cave Bei geriatrischen Patienten sind klinische Exsikkosezeichen nicht sehr sensitiv [22].

Labordiagnostik | Wenn laborchemische Untersuchungen verfügbar sind, deuten folgende Parameter auf eine Exsikkose:

- erhöhte Nierenretentionswerte,

- erhöhter Hämatokrit,

- Hypernatriämie.

Diese Befunde müssen aber immer im klinischen Kontext beurteilt werden.

Fremdbeobachtung I Bei eingeschränkter Anamnesefähigkeit, z.B. im Rahmen einer Demenz, ist eine Fremdbeobachtung hilfreich. Diese wird am sichersten durch Angehörige oder pflegende Personen erhoben. Dabei sollte eine zunehmende Lethargie und Desorientiertung erfragt werden.

\section{Therapiemöglichkeiten}

Erhalt der Flüssigkeitshomöostase | Wenn klinische Zeichen oder Laborwerte auf eine Exsikkose bzw. einen Volumenmangel schließen lassen, ist eine Rehydration indiziert. Gleichzeitig sollte die Dauermedikation überprüft und ggf. eine antidiuretische Therapie reduziert werden. Der Flüssigkeitshaushalt muss im Therapieverlauf wiederholt klinisch kontrolliert werden.

Orale Rehydratation | Die orale Flüssigkeitszufuhr ist die komplikationsärmste, aber auch zeitintensivste Form der Rehydratation. Soweit keine komplizierte Schluckstörung vorliegt, ist sie die Therapie der Wahl. Umgesetzt werden kann das Verfahren z.B. durch

- Anwendung von Trinkplänen

- wiederholtem Anbieten von Getränken durch Angehörige oder Pflegepersonen [24].

Die freie Verfügbarkeit von Wasser und Tee in professionellen Pflegeeinrichtungen bedeutet jedoch noch lange keine ausreichende Flüssigkeitsaufnahme. Neben Schulung und Bewusstseinsbildung sollte eine ausreichende Stellenbesetzung in Pflegeeinrichtungen angestrebt werden. In der häuslichen Pflege ist eine Schulung der Angehörigen zum Umgang mit Trinkplänen anzubieten.

„Lieblingsgetränke“ können die

Therapieadhärenz bei oraler Rehydratation erhöhen und Krankenhauseinweisungen vermindern [35].

Subkutane Rehydratation (Hypodermoclysis) I Die seit ca. 150 Jahren praktizierte Methode der Hypodermoclysis ist in der Geriatrie ein etabliertes Verfahren, das im stationären Bereich auch längerfristig angewendet wird [17]. Das Verfahren wird in der S3-Leitlinie der Deutschen Gesellschaft für Ernährungsmedizin bei geriatrischen Patienten mit leichter bis mittelschwerer Exsikkose empfohlen [6]. Ebenso wie die kaum noch praktizierte rektale Flüssigkeitsgabe $[9,10]$ kann die Hypodermoclysis auch von Pflegepersonal und geschulten Laien angewandt werden.

Intravenöse Rehydratation | Die Hypodermoclysis ist eine sichere und kostengünstige Alternative zur intravenösen Flüssigkeitsgabe. Für schwere Exsikkose- und Schockzustände ist allerdings eine intravenöse Substitution notwendig.

Rehydratation über eine Sonde I Falls eine Ernährung oder Flüssigkeitsaufnahme über eine vorhandene perkutane Sonde erfolgt, sollte ein erhöhter Flüssigkeitsbedarf über die Sonde ausgeglichen werden [6]. 


\section{Indikationen}

leichte bis mittelschwere Exsikkose bei

- Dysphagie (z. B. bei Soor)

- Erbrechen/Diarrhoe

- virale Infekte

- Harnwegsinfekte

- schlechtem Venenstatus

- Delir bei Exsikkose

- Hitzeperioden

\section{Kontraindikationen}

schwere Dehydratation

Schock

Sepsis

Volumenüberladung

notwendige intravenöse Medikation

starke Blutungsneigung bei Gerinnungsstörungen /

Antikoagulation
Tab. 1 Indikationen und Kontraindikationen der Hypodermoclysis

\section{Anwendung der Hypodermoclysis}

Indikation | Nach einem Beschluss des Gemeinsamen Bundesausschusses (G-BA) kann die Hypodermoclysis seit August 2013 im Rahmen der häuslichen Krankenpflege verordnet werden (BAnz 20.08.2013 B3). Das Verfahren sollte vermehrt angewendet werden zur

- Therapie von leichten bis mittelschweren Dehydratationszuständen,

- Prävention von Exsikkosezuständen [31], auch wenn dies im aktuellen Beschluss des G-BA nicht vorgesehen ist.

- Tab. 1 fasst Indikationen und Kontraindikationen zusammen.

Ambulante Anwendung I Der G-BA betont eine enge Indikationsstellung bei der Anwendung im ambulanten Bereich. So darf die Infusion nur nach sorgfältiger Abwägung durch den behandelnden Arzt verordnet und delegiert werden. Dies ist gebunden an eine vorherige persönliche Überzeugung über den Zustand des betroffenen Patienten. Die Verordnungsfähigkeit wurde auf 7 Tage begrenzt, unter der Prämisse einer regelmäßigen Rücksprache zwischen Arzt und dem ausführenden Pflegepersonal.

\section{Technik der Hypodermoclysis}

Materialien | Die Flüssigkeit wird über ein beliebiges handelsübliches Infusionsset in die Subkutis eingebracht.

- Zugang: Butterfly-Nadel (Stahl), Teflon- oder Vialon-Katheter [20, 31]

- Durchmesser: 20-28 G [12, 42, 44]

- Liegedauer: üblicherweise 24-72 Stunden [42]. Durch die Benutzung von Teflon- oder Vialon-Kathetern kann die Nutzungsdauer auf max. 12 Tage erhöht werden [20, 31]. In dieser Zeit können mehrere Infusionen über eine Nadel verabreicht werden.

Durch Teflon- oder Vialon-Katheter können Verletzungen vermieden und die Nutzungsdauer erhöht werden.
Vorgehensweise I Die Infusionsnadel wird unter sterilen Bedingungen nach lokaler hygienischer Hautdesinfektion in einem $45^{\circ}$-Winkel in die Subkutis eingebracht. Dazu wird eine Hautfalte wie bei einer subkutanen Injektion angehoben [12, 36, 45]. In 24 Stunden könnenbis zu 31 Volumen verabreicht werden (max. 1,51 pro Applikationsort). Die Flussgeschwindigkeit liegt bei max. $250 \mathrm{ml} / \mathrm{h}$, nach Fachinformationen bei $1-2 \mathrm{ml} / \mathrm{min}[6,12,17]$. Eine Fixierung des Katheters mit einem transparenten Verband erlaubt es, die Einstichstelle beurteilen zu können $[18,44]$.

Infusionsorte | Geeignete Applikationsorte sind

- die laterale Bauchwand,

- Oberschenkel und

- die Subclavia-Region.

Bei unruhigen, desorientierten Patienten kann auch die Gabe am Rücken, zwischen den Schulterblättern, gewählt werden. Die Kanüle wird dort selten entfernt, da sie weniger sichtbar und schlechter erreichbar ist $[6,12,18,36]$.

Cave Um bei einer intraskapulären Applikation Verletzungen der Lunge zu vermeiden, sollte der Patient sitzen. Verwenden Sie keine Stahlkanüle.

Infusionslösungen | Bei der Behandlung von Volumenmangel und Exsikkose sollten Elektrolytlösungen verwendet werden. Zugelassen sind:

- 0,9\%-ige NaCl-Lösung,

- Tutofusin (Vollelektrolytlösung; Baxter),

- Jonosteril ${ }^{\circledR}$ (Vollelektrolytlösung; Fresenius).

Die Auswahl sollte in Anbetracht vorhandener Laborwerte oder Begleiterkrankungen getroffen werden:

- $\mathrm{NaCl}$ 0,9\% zur kaliumarmen Substitution bei Patienten mit Nierenfunktionsstörungen und Neigung zur Hyperkaliämie [6, 12, 30, 37, 38],

- Vollelektrolytlösungen zur kurzzeitigen Substitution bei febrilen Infekten, Diarrhö oder intensivierter Diurese. Da hier von einem Elektrolytverlust im Rahmen der verursachenden Problematik auszugehen ist, ist keine Laborwertkontrolle nötig. 


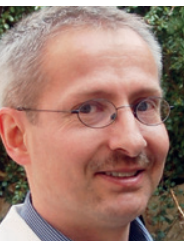

Dr. med. Joachim Schnürle ist Leiter des Fachbereichs Innere Medizin und Hygienebeauftragter Arzt der Altmühlseeklinik Hensoltshöhe, Gunzenhausen. joaschnuerle@hotmail.com
Elektrolytfreie Glukoselösung kann durch osmotische Umverteilungen zu fatalen Ausgängen führen und sollte nicht eingesetzt werden [1, 2].

\section{Komplikationen bei Hypodermoclysis}

In den USA wurden in den 50er Jahren des 20. Jahrhunderts Komplikationen berichtet, die retrospektiv auf eine ungeeignete Indikationsstellung zurückzuführen waren [2]. Seit den 90er Jahren wurden zahlreiche Studien zur Sicherheit des Verfahrens veröffentlicht [17, 26, 30, 36, 38].

Lokale Reaktionen I Üblicherweise kommt es zu einer lokalen Schwellung, die bis zu 12 Stunden anhält, da die subkutane Flüssigkeit verzögert resorbiert wird. Dies ist jedoch nicht als Komplikation anzusehen [44, 45]. Zu den lokalen Komplikationen gehören Hauteinblutungen und subkutane Inflammation. Rötungen um die Einstichstelle treten meist später als 72 Stunden nach Anlage der Nadel auf [43].

Durch eine tägliche Inspektion der Applikationsstelle können lokale Komplikationen frühzeitig erkannt werden. Die Entfernung des Katheters reicht meist aus, um die Entzündung zu behandeln.

Hyperhydratation I Komplikationen wie eine Überwässerung wurden beschrieben. Dies ist aber kein spezifisches Problem der Methode, sondern vielmehr ein Hinweis auf eine falsche Indikationsstellung oder zu hohe Dosierung der Flüssigkeitsgabe. Hyperhydrationszustände können Sie ausschließen durch

- Gewichtskontrollen,

- Befragung nach zunehmender Atemnot und

- Inspektion auf periphere Ödem.

Ödembildung | Frühere Empfehlungen, eine Ödembildung durch Gabe von Hyaluronsäure zu vermeiden, werden heute nicht routinemäßig gegeben. 150IE Hyaluronsäure je $1000 \mathrm{ml}$ Infusion vermindert die interstitielle Viskosität für 24 bis 48 Stunden, verbessert aber nicht die Resorption $[30,36]$. Zudem besteht die Gefahr allergischer Reaktionen auf Hyaluronsäure [18].

2008 wurde bei einer kachektischen Patientin eine Zökumperforation berichtet. Dies zeigt, dass eine vorsichtige Anlage des Katheters nach sorgfältiger Auswahl des Applikationsortes nötig ist [25]. intravenösen Applikation. In Studien wurde darauf hingewiesen, dass bei schlechten Venenverhältnissen wiederholte Venenpunktionen einen erhöhten Materialbedarf erzeugen, was bei der subkutanen Infusion entfällt [4].

Personalkosten I Die Personalkosten sind bei der Hypodermoclysis deutlich geringer als bei der intravenösen Substitution. Die Infusion erfolgt durch Pflegepersonal und kann prinzipiell auch von geschulten Laien durchgeführt werden.

Behandlungskosten | Die weitaus meisten Kosten können allerdings dadurch eingespart werden, dass Krankenhauseinweisungen vermieden werden. In Deutschland belaufen sich die Kosten einer ambulanten subkutanen Rehydratation auf $18 €$ vs. $485 €$ pro Tag bei stationärer intravenöser Substitution [44]. Für das amerikanische Gesundheitssystem wurden bei einer Liegedauer von 4 bis 6 Tagen vermeidbare stationäre Kosten von über einer Milliarde Dollar berechnet [27, 42].

Vermeidung von Krankenhausaufenthalten I Nicht selten ist eine Krankenhauseinweisung für betagte Menschen der Eintritt in eine gesundheitliche Abwärtsspirale - für manche der „Anfang vom Ende“. Auch wenn in den letzten Jahren zunehmend kurzstationäre Maßnahmen eingeführt wurden [13], sollten neben der Kostenseite die potenziellen Gefahren einer Krankenhauseinweisung bedacht werden, $\mathrm{z}$. B.

- Entwicklung von Verwirrtheitszuständen und deliranten Episoden [19, 34],

- Stürze und Frakturen,

- nosokomiale Infektionen.

\section{Konsequenz für Klinik und Praxis}

- Während Hitzeperioden, bei febrilen Infekten und unter Diuretika müssen Trinkverhalten und Flüssigkeitsstatus von Pflegebedürftigen genau beobachtet werden.

- Bei Anzeichen für eine Exsikkose sollte eine diuretische Therapie pausiert werden.

- Wenn eine orale Flüssigkeitsaufnahme nicht gewährleistet werden kann, ist eine subkutane Rehydratation anzustreben.

- Während einer Substitutionstherapie muss eine tägliche Begutachtung des Patienten erfolgen, um die Indikation zu überpüfen.

- Die subkutane Flüssigkeitsgabe kann vom Hausarzt verordnet und ambulant durch einen Pflegedienst durchgeführt werden.

- Durch eine subkutane Rehydratation können Krankenhauseinweisungen vermieden und das stationäre Gesundheitswesen entlastet werden.

\section{Vorteile der Hypodermoclysis}

Materialkosten | Die Materialkosten für die subkutane Flüssigkeitsgabe sind vergleichbar mit der
Vollständiges Literaturverzeichnis unter http://dx.doi.org/10.1055/s-0041-102093 


\section{Literatur}

1 Abbott WE, Levey S, Foreman RC et al. The danger of administering parenteral fluids by hypodermoclysis. Surgery 1952; 32: 305-315

2 Barua P, Bhowmick BK. Hypodermoclysis - a victim of historical prejudice. Age and Ageing 2005; 34: 215-217

3 Begley S. Are you ready for more? In a world of climate change, freak storms are the new normal. Why we're unprepared for the harrowing future. Newsweek 2011; June 6th: 28-34

4 Berger EY. Nutrition by Hypodermoclysis. I Am Geriatr Soc 1984; 32: 199-203

5 Bhave G, Neilson EG. Volume depletion versus dehydration: How understanding the difference can guide therapy. Am J Kidney Dis 2011; 58: 302-309

6 Bischoff SC, Arends J, Dörje F et al. Künstliche Ernährung im ambulanten Bereich. Aktuel Ernahrungsmed 2013; 38: e101-e154

7 Bruera E, Belzile M, Watanabe S et al. Volume of hydration in terminal cancer patients. Support Care Cancer 1996; 4: 147-150

8 Bruera E, Legris MA, Kuehn $\mathrm{N}$ et al. Hypodermoclysis for the administration of fluids and narcotic analgesics in patients with advanced cancer. J Pain Symptom Manage 1990; 5: 218-220

9 Bruera E, Pruvost M, Schoeller T et al. Proctoclysis for Hydration of Terminally III Cancer Patients. J Pain Symptom Manage 1998; 15: 216-219

10 Daland J. The treatment of Cholera by hypodermoclysis and enteroclysis. Trans Am Climatolog Clin Assoc 1893; 10: $92-104$

11 D'lppoliti D, Michaelozzi P, Marino C et al. The impact of heat waves on mortality in 9 European cities: results from the EuroHEAT poject. Environ Health 2010; 9: 37

12 Ferry M, Dardaine V, Constans T. Subcutaneous Infusion or Hypodermoclysis: A practical approach. J Am Geriatr Soc 1999; 47: 93-95

13 Groening M, Schwarz T, Lock G. Versorgung älterer Notfallpatienten: Hightouch statt Hightech. Dtsch Arztebl 2013; 110: A 262-265

14 Hansen A, Bi P, Nitschke M et al. Perceptions of Heat-Susceptibility in Older Persons: Barriers to Adaption. Int J Environ Res Public Health 2011; 8: 4714-4728

15 Kayser-Jones ], Schell ES, Porter C et al. Factors contributing to dehydration in nursing homes: inadequate staffing and lack of professional supervision. J Am Geriatr Soc 1999; 47: 1187-1194

16 Kenney WL, Munce TA. Physiology of Aging, Invited Review: Aging and human temperature regulation. J Appl Physiol 2003; 95: 2598-2603

17 Leischker $\mathrm{AH}$. Subkutane Flüssigkeitsgabe für geriatrische Patienten: Eine „alte“ Technik wird wiederentdeckt. Z Gerontol Geriatr 2012; 45: 665-672

18 Lopez JH, Reyes-Ortiz C. A Subcutaneous hydration by hypodermoclysis. Rev Clin Gerontol 2010; 20: 105-113

19 Lorenzl S, Füsgen I, Noachtar S. Verwirrtheitszustände im Alter: Diagnostik und Therapie. Dtsch Arztebl Int 2012; 109: 391-400

20 Macmillan K, Bruera E, Kuehn N et al. A prospective comparison study between a butterfly needle and a Teflon cannula for subcutaneous narcotic administration. J Pain Symptom Manage 1994; 9: 82-84

21 Maughan RJ. Hydration, morbidity, and mortality in vulnerable populations. Nutr rev 2012; 70: S152-S155

22 McGee S, Abernethy WB 3 rd, Simel DL. Is this patient hypovolemic? JAMA 1999; 281: 1022-1029

23 Meehl GA, Tebaldi C. More intense, more frequent, and longer lasting heat waves in the 21 st century. Science 2004; 305: 994-997

24 Mentes J. Oral hydration in older adults. AJN 2006; 106 : 40-49
25 Mongardon N, Le Manach Y, Lescot T et al. Subcutaneous hydration: a potentially hazardous route. Eur J Anesthesiol 2008; 25: 771-72

26 O' Keeffe ST, Lavan JN. Subcutaneous fluids in elderly hospital patients with cognitive impairment. Gerontology 1996; 42: 36-39

27 Pershad J. A systematic data review of the cost of rehydration therapy. Appl Health Econ Health Policy 2010; 8: 203-214

28 Philipps PA, Rolls BJ, Ledingham JGG. Reduced thirst after water deprivation in healthy elderly men. N Engl J Med 1984; 311: 753-756

29 Remington R, Hultman T. Hypodermoclysis to Treat Dehydration: A Review of Evidence. J Am Geriatr Soc 2007; 55: 2051-2055

30 Rochon PA, Gill SS, Litner J et al. A systematic review of the evidence for hypodermoclysis to treat dehydration in older people. J Gerontol A Biol Sci Med Sci 1997; 52: M169-M176

31 Ross JR, Saunders Y, Cochrane M et al. Prospective, within-patient comparison between metal butterfly needles and Teflon cannulae in subcutaneous infusion of drugs to terminally ill hospice patients. Palliat Med 2002; 16: 13-16

32 Schnieders M, Kolb G. Exsiccose im Alter. Med. Klin 2004; 99: 453-460

33 Schols JM, De Groot CP, van der Cammen TJ et al. Preventing and treating dehydration in the elderly during periods of illness and warm weather. J Nutr Health Aging 2009; 13: 150-157

34 Seymour DG, Henschke PJ, Cape RDT et al. Acute confusional states and dementia in the elderly: The role of Dehydration / Volume depletion, physical illness and age. Age Ageing 1980; 9: 137-146

35 Simmons SF, Alessi C, Schnelle JF. An Intervention to increase Fluid intake in Nursing home Residents: Prompting and Preference Compliance. J Am Geriatr Soc 2001; 49: 926-933

36 Slesak G, Schnürle JW, Kinzel E et al. Comparison of Subcutaneous and Intravenous Rehydration in Geriatric Patients: A Randomized Trial, J Am Geriatr Soc 2003; 51: $155-160$

37 Thomas DR, Cote TR, Lawhorne L et al. Understanding Clinical Dehydration and Its Treatment. J Am Med Dir Assoc 2008; 9: 292-301

38 Turner T, Cassano AM. Subcutaneous dextrose for rehydration of elderly patients - an evidence-based review. BMC Geriatr 2004; 4: 2

39 United Nations Environmental Programme DEWA/RID - Europe: Impact of summer 2003 in Europe. Environmental Alert Bulletin 2004; 2

40 Von Wichert P. Gefährdung durch atmosphärische Hitzewellen. Mitteilungen AWMF 2004; 1: Doc35

41 Von Wichert P. Klimatische Hitzewellen und deren Konsequenzen für die gesundheitliche Betreuung vorgeschädigter Personen. Med Klin 2008; 103: 75-79

42 Xiao H, Barber J, Campbell ES. Economic burden of dehydration among hospitalized elderly patients. Am J Health Syst Pharm 2004; 61: 2534-2540

43 Yap LK, Tan SH, Koo WH. Hypodermoclysis or Subcutaneous Infusion Revisited. Singapore Med J 2001; 42: 526-529

44 Zeeh J. Exsikkose im Alter - vom Umgang mit Patienten, die zu wenig trinken-Stellenwert der subkutanen Flüssigkeitsgabe (Hypodermoclyse). Ärzteblatt Thüringen 2009; 20: 256-259

45 Zeeh J, Pöltz S. Subkutane Flüssigkeitszufuhr, elegant, sicher und effektiv. Geriatrie Journal 2000; 8: 28-30 